Luis ROSERO-BIXBY****, Teresa CASTRO-MARTíN ${ }^{\circ} * *$, David REHER ${ }^{\nu_{*} * *}$ et María SÁNCHEZ-DOMÍNGUEZ ${ }^{\vee_{*} * *}$

\title{
Estimation indirecte du nombre d'immigrés en Espagne à partir des taux de fécondité et des naissances
}

\begin{abstract}
Certaines caractéristiques de la population peuvent être estimées de plusieurs manières, et la confrontation des différentes estimations permet de comparer les méthodes et de mieux comprendre les limites de chacune d'entre elles. Depuis 1996, l'Espagne dispose d'un registre de population regroupant tous les registres municipaux ; il inclut l'ensemble des immigrants, permettant ainsi l'évaluation directe de leur nombre dans sa population. Par ailleurs, une enquête de 2007 permet d'estimer la fécondité des immigrants en Espagne, par sexe et âge. Dans cet article, Luis Rosero-BIXBY, Teresa CASTRO-MARTín, David ReHER et María SÁNCHEZ-DOMínGUEZ partent des taux de fécondité des immigrants issus de l'enquête et du nombre de naissances disponible dans l'état civil pour déduire le nombre d'immigrants en Espagne par pays d'origine. La comparaison des résultats avec les effectifs présents dans les registres leur permet de mettre la méthode à l'épreuve et de préciser les limites du registre, en particulier la surestimation probable du nombre d'hommes immigrants.
\end{abstract}

L'Espagne, pays d'émigration pendant des siècles, est devenu pays d'immigration depuis deux décennies (Arango, 2000 ; Cebolla et González-Ferrer, 2008). D’après les données du recensement et du registre de population, l'Espagne accueillait 350000 étrangers en 1991, 1,5 million en 2001 et 5,7 millions en 2011, le poids relatif des étrangers dans la population totale étant passé de 0,9\% en 1991 à 12,2 \% en 2011, proportion comparable à celle observée dans

\footnotetext{
* Universidad de Costa Rica.

- Centro de Ciencias Humanas y Sociales, CSIC, Espagne.

$\checkmark$ Universidad Complutense de Madrid.

** Grupo de Estudios Población y Sociedad, GEPS, Espagne.

Correspondance : Luis Rosero-Bixby, Centro Centroamericano de Población, Universidad de Costa Rica, San Pedro 2060, San Jose, Costa Rica, courriel: lrosero@mac.com
} 
des pays ayant une tradition d'immigration beaucoup plus ancienne. Depuis 2000, l'Espagne a accueilli environ le tiers des migrants entrant dans l'Union européenne (Eurostat, 2009), même si la crise économique récente a réduit la plupart des flux migratoires (Martin, 2009 ; Reher et al., 2011b). L'une des conséquences en est la contribution croissante à la dynamique démographique (Izquierdo et López de Lera, 2006). Au cours de la dernière décennie, la migration nette a contribué approximativement à $90 \%$ de la croissance de la population espagnole (Roig et Castro Martín, 2007). En 2009, 20,7 \% de l'ensemble des naissances vivantes étaient issues de mères étrangères, et 23,9 \% de pères ou mères étrangers.

Cet article a pour objectif de fournir des estimations indépendantes du nombre d'immigrés en Espagne en « suivant leurs traces », notamment en utilisant les informations sur le nombre de naissances selon l'origine des parents enregistrées par l'état civil. Nos estimations combinent ces informations avec celles sur les taux de fécondité des immigrés calculés à partir de l'Enquête nationale sur les immigrés de 2007 (Encuesta Nacional de Inmigrantes, ENI). Si nos estimations ne diffèrent pas du décompte des immigrés dans le registre municipal de population espagnol, ce sera une validation importante à la fois du registre, de la procédure d'estimation et des données utilisées.

Le registre municipal de population espagnol (padrón municipal) est un registre administratif dans lequel sont inscrits tous les résidents d'une commune, quels que soient leur citoyenneté ou leur statut légal de résidence. Ces listes sont créées et mises à jour par les administrations municipales. Depuis 1996, un nouveau système de gestion continue et informatisée a été introduit pour tous les registres municipaux, coordonné par l'Institut national de statistique (Instituto Nacional de Estadística, INE), qui effectue les vérifications permettant de corriger les erreurs et d'éliminer les doubles comptes, et qui génère un fichier centralisé. À partir de celui-ci, les effectifs officiels de population sont établis chaque année pour les communes, les provinces, les communautés autonomes et le pays dans son ensemble.

Ce registre de population constitue une source d'information sur la population immigrée plus fiable que d'autres sources, comme l'annuaire étranger du ministère de l'Intérieur qui ne couvre que les immigrés titulaires d'une autorisation de résidence en règle. De nombreux étrangers n'ont pas les documents nécessaires pour résider et travailler légalement en Espagne (Moreno, 2005). Par exemple, début 2009, environ 4,5 millions d'étrangers avaient une autorisation de résidence en cours de validité, soit 1,1 million de moins que le nombre d'étrangers comptabilisés dans le registre de population ${ }^{(1)}$. Lors de la dernière campagne de régularisation mi-2005, 560000 immigrés sans papiers

(1) Seul un tiers de cet écart est dû à la présence de citoyens de l'Union européenne, qui n'ont pas besoin d'autorisation de résidence. Le pourcentage total d'immigrés vivant en Espagne sans cette autorisation a été estimé à 70 \% de l'ensemble de la population étrangère en 2002, 40 \% en 2005 et $24 \%$ en 2007 (Cebolla et González Ferrer, 2008). 
ont obtenu un titre de séjour à condition d'avoir un contrat de travail, mais il n'y a pas eu d'autre programme de régularisation exceptionnelle depuis ${ }^{(2)}$.

On considère que la couverture des registres municipaux de population est élevée, puisque le fait d'y être inclus donne un accès automatique aux services d'enseignement et de santé( ${ }^{(3)}$, et qu'il est une condition préalable à l'obtention d'une autorisation de résidence légale pour les personnes qui ne la détienne pas à leur arrivée. En fait, l'Espagne est le seul pays européen qui autorise et favorise l'enregistrement des immigrés irréguliers dans un registre de population (González-Enríquez, 2009) et on peut penser que le décompte de la population immigrée y est plus complet qu'ailleurs. Cependant, les registres municipaux de population ne sont sans doute pas sans défaut. Malgré les encouragements à s'enregistrer, plusieurs recherches font apparaître que certains groupes - en particulier les employés des travaux agricoles saisonniers ou ceux craignant d'être expulsés - sont sous-estimés, et qu'un intervalle sépare l'arrivée dans le pays et l'enregistrement (Devolder et al., 2003). Un sous-enregistrement est également vraisemblable parmi les enfants, mais il recule aux âges pour lesquels l'inscription à l'école nécessite un certificat du registre municipal de population. À l'inverse, des études mettent en garde contre une certaine surestimation du nombre réel d'immigrés par le registre de population (Ródenas et Martí, 2009). Ce peut être le cas à cause de doubles enregistrements difficiles à détecter pour les résidents étrangers utilisant plusieurs documents d'identité dans leurs démarches administratives ${ }^{(4)}$, ou parce que les immigrés omettent généralement de se faire radier quand ils retournent dans leur pays d'origine ou qu'ils émigrent vers un autre pays. Étant donné que le montant des financements alloués par le gouvernement central aux municipalités dépend en partie des effectifs de population, il est traditionnellement plus facile de s'inscrire dans le registre municipal de population que d'en être radié. L'inscription est encouragée et comporte des intérêts visibles pour le bénéficiaire, alors qu'il n'y a pas d'incitation à la radiation. Comme souvent dans les registres administratifs, la détection et l'élimination des erreurs sont extrêmement difficiles et celles-ci peuvent devenir une composante structurelle du système. Pour pallier ce problème, une réforme législative (14/2003, 20 novembre 2003) a introduit une modification importante : les étrangers hors Union européenne

(2) Des programmes spécifiques de régularisation ont été instaurés en 1986, 1991, 1996, 2000-2001 et 2005. Ils ont accordé des titres de séjour temporaires et ont permis à une proportion importante d'immigrés de passer de l'économie informelle au marché du travail officiel. Au total, 1,1 million d'immigrés ont bénéficié de ces régularisations, dont 52 \% en 2005 (González-Enríquez, 2009).

(3) Depuis 2000, tous les étrangers inclus dans un registre municipal, quel que soit leur statut légal, bénéficient du libre accès à l'enseignement public de 3 à 16 ans et au service public de santé. Les étrangers hors registre n'ont accès qu'aux services d'urgence, à l'exception des mineurs et des femmes enceintes. Pour s'inscrire dans le registre il suffit d'une preuve d'identité (carte d'identité espagnole, titre de séjour ou passeport) et d'une preuve de résidence à l'adresse déclarée (titre de propriété, contrat de location, facture de gaz, électricité, etc., ou une lettre du premier adulte déjà enregistré à cette adresse).

(4) Par exemple, un résident étranger peut s'enregistrer d'abord avec son numéro de passeport, puis avec son numéro de titre de séjour dans une autre commune. 
qui ne détiennent pas de titre de séjour permanent doivent désormais renouveler leur inscription au registre de population tous les deux ans, sous peine d'être radiés d'office. Cette mesure a certainement réduit le surenregistrement à partir de 2006, deux ans après l'entrée en vigueur de la nouvelle procédure $^{(5)}$.

Dans le riche arsenal des techniques de mesures indirectes en démographie, la plupart des méthodes sont consacrées à l'étude de la mortalité et de la fécondité. À titre d'exemple, le manuel classique des Nations unies sur les techniques indirectes d'estimation en démographie (Nations unies, 1983) ignore complètement la migration. Un groupe de travail de l'UIESP s'est consacré aux méthodes indirectes dans l'étude de la migration internationale, mais il a privilégié les flux d'émigration (Zaba, 1985). Il existe toutefois quelques exemples d'utilisation de techniques indirectes pour estimer le nombre d'immigrés illégaux aux États-Unis, s'appuyant sur des informations à propos des rapports de masculinité (Bean et al., 1983), des taux de mortalité (Robinson, 1980 ; Borjas et al., 1991) ou ceux de scolarité (Muller et Espenshade, 1985). Le présent article prolonge un travail précédent d'estimation du nombre de Nicaraguayens au Costa Rica (Rosero-Bixby et al., 2002) et propose d'estimer le nombre d'immigrés en Espagne à partir de données sur les naissances enregistrées dans l'état civil et les taux de fécondité mesurés par enquête. Cette méthode pourrait être étendue à un large éventail de situations.

\section{Données et méthodes}

Le nombre de naissances issues de parents immigrés est notre point de départ. Dans cette étude, les immigrés sont définis comme des personnes résidant en Espagne mais nées à l'étranger, quels que soient leur statut légal, leur nationalité à la naissance et leur nationalité actuelle. Alors que les données sur les naissances par nationalité des parents sont disponibles librement sur le site internet de l'Institut national de statistiques depuis 1996 (www.ine.es), le classement par pays de naissance des parents n'est disponible que depuis 2007. Nous avons choisi de fonder notre analyse sur le pays de naissance plutôt que sur la nationalité afin d'éviter d'éventuelles divergences entre les sources de données, notamment à cause du processus de naturalisation (GonzálezFerrer et Cortina, 2011). La couverture des statistiques de naissances est considérée comme complète en Espagne et il n'y a pas de raison de croire que l'enregistrement des naissances d'enfants d'immigrés ne le soit pas.

La méthode proposée requiert également une estimation des taux de fécondité des immigrés, afin d'utiliser le nombre des naissances pour faire une nouvelle estimation de la population dont elles sont issues. Nous avons estimé les taux de fécondité des immigrés à partir des données de l'Enquête nationale sur les

(5) Environ 250000 étrangers ont été radiés d'office en 2006, suite à la nouvelle loi (GonzálezFerrer, 2009). 
immigrés (ENI), réalisée par l'Institut national de statistiques en 2007 (INE, 2009). L'échantillon visait les personnes nées à l'étranger et âgées de 16 ans ou plus, qui avaient résidé en Espagne au moins un an au moment de l'enquête, ou qui avaient l'intention de résider en Espagne au moins un an, quel que soit leur statut légal ${ }^{(6)}$. Un plan de sondage à trois degrés a permis de sélectionner des logements dans lesquels résidait au moins un étranger. Les unités de premier niveau étaient les sections de recensement, groupées en strates en fonction de la taille de la commune. La sélection des sections a suivi une probabilité proportionnelle au nombre d'étrangers éligibles. Les unités de second niveau étaient des ménages privés, qui présentaient la même probabilité de tirage par un échantillonnage systématique, après avoir été classés en fonction de la nationalité prédominante. Au troisième niveau, l'unité était un individu sélectionné avec une probabilité égale dans une liste de personnes nées à l'étranger résidant dans chaque logement (INE, 2008). Au total, 15465 questionnaires ont été validés à l'issue du travail de terrain, suite à la visite de 32541 logements. Parmi les logements dans lesquels résidait au moins une personne née à l'étranger et qui étaient donc éligibles pour l'entretien (17 700), le taux de réponse a été de 87,4\%. Des coefficients de pondération corrigent le suréchantillonnage de certaines régions et les différences des taux de non-réponse par âge, sexe et région. Des informations supplémentaires sur la méthodologie de l'enquête et la fiabilité des données sont publiées par INE (2008) ainsi que Reher et Requena (2009a). Les données de l'enquête ont déjà été utilisées dans de nombreuses publications concernant l'immigration en Espagne ${ }^{(7)}$.

Les estimations de la fécondité à partir des histoires génésiques dans les enquêtes démographiques rétrospectives tendent souvent à surestimer le niveau réel à cause de taux de non-réponse plus élevé pour les femmes sans enfant, qui sont généralement plus difficiles à joindre chez elles pour un entretien (Festy et Prioux, 2002). Dans l'ENI, ce biais potentiel était réduit par la possibilité laissée à un autre membre du ménage de fournir les informations concernant les individus difficiles à atteindre. Le biais existe cependant pour les ménages d'une seule personne et pour les personnes ayant des liens ténus avec le reste du ménage. On notera que le taux de non-réponse de 12,6\% parmi les personnes éligibles se situe bien dans la fourchette de 7-20\%, considérée par Festy et Prioux comme un niveau de qualité acceptable pour les données d'enquête sur la fécondité (2002, p. 19).

Nous avons traité les microdonnées de l'enquête avec le logiciel Stata-10 pour estimer les taux de fécondité (Statacorp, 2007). Bien que la taille importante de l'échantillon de l'ENI permette d'obtenir des estimations fiables pour l'ensemble de la population immigrée et certains grands groupes définis selon

(6) $11,2 \%$ des répondants ayant besoin d'une autorisation de résidence (puisque ni citoyens, ni membres de l'Union européenne) n'en détenaient pas, et 6,5\% ne l'avaient pas encore reçue.

(7) Voir par exemple Reher et Silvestre (2009), ainsi que les articles publiés dans Reher et Requena (2009b) et dans Reher et al. (2011a). 
l'origine, des erreurs d'échantillonnage dans les taux de fécondité par âge limitent le niveau de détail auquel nous pouvons travailler et introduisent des incertitudes non négligeables dans nos estimations. Dans les analyses exploratoires des niveaux de fécondité et des profils par âge, nous avons procédé à un lissage des taux de fécondité par année d'âge des immigrés classés par groupes de pays de naissance. Cependant, pour estimer le nombre d'immigrés, nous avons utilisé des taux quinquennaux de fécondité entre 25 et 35 ans pour les femmes, entre 30 et 40 ans pour les hommes, en excluant les âges extrêmes auxquels les taux calculés à partir de l'enquête n'étaient pas fiables. Dans toutes nos estimations de la fécondité nous avons utilisé les pondérations fournies par la base de données de l'ENI. En plus des habituels taux de fécondité féminins, nous avons estimé les taux de fécondité des hommes immigrés.

À partir de la série des taux de fécondité par âge, nous avons utilisé la formule suivante pour estimer le nombre d'immigrés et d'immigrées d'âge fécond I provenant du pays ou de la région $i$ :

$$
I^{i}=\sum_{a} \frac{N_{a}^{i}}{f_{a}^{i}}
$$

où :

$\mathrm{N}$ est le nombre de naissances issues de pères ou de mères par pays d'origine $i$ et appartenant au groupe d'âges a (selon les données d'état civil);

$f$ est le taux de fécondité des immigrés par pays d'origine $i$, dans le groupe d'âges $a$ (estimations par sexe à partir de l'enquête ENI).

Afin de prendre en compte l'hétérogénéité de la population immigrée, la procédure d'estimation a été conduite séparément pour les pays les plus représentés en Espagne (Maroc, Équateur et Roumanie) et pour les groupes importants de pays. Nous avons d'abord déterminé 11 groupes de pays des immigrés par origine (tableau 1) à condition que les échantillons de chaque groupe comptabilisent au moins 200 observations. Les modèles de régression n'ayant pas fait apparaître de différence significative entre certains de ces groupes pour les niveaux et profils de fécondité, nous avons finalement ramené les pays d'origine à 7 groupes par la suite : (1) Équateur, (2) Autres pays d'Amérique latine, (3) Maroc, (4) Autres pays d'Afrique, (5) Europe occidentale, (6) Roumanie et (7) Europe orientale et Asie ${ }^{(8)}$.

Pour estimer les taux de fécondité de la période 2004-2006 (les trois années ayant précédé l'ENI), nous avons construit l'histoire génésique complète de chaque répondant âgé de plus de 15 ans ${ }^{(9)}$ grâce à l'information disponible dans l'enquête pour les trois groupes d'enfants suivants :

(8) L'échantillon d'Asiatiques étant très limité (environ 100 femmes et 200 hommes), l'ENI n'a pas la capacité d'identifier des différences significatives en matière de fécondité ; nous avons donc dû fondre ce groupe dans un autre. Les immigrants d'Europe de l'Est constituent la population la plus proche pour ce regroupement.

(9) L'ENI a interrogé des immigrés âgés de 16 ans ou plus. On peut cependant estimer les taux à 15 ans de la période 2004-2006 grâce aux histoires génésiques des répondants âgés de 16 à 18 ans. 
Tableau 1. Effectifs de l'échantillon ENI utilisés pour l'estimation des taux de fécondité et des nombres d'immigrés

\begin{tabular}{|c|c|c|c|c|}
\hline \multirow[b]{2}{*}{ Pays de naissance } & \multicolumn{2}{|c|}{ Pour les taux de fécondité } & \multicolumn{2}{|c|}{ Pour les nombres d'immigrés } \\
\hline & $\begin{array}{l}\text { Femmes } \\
16-44 \text { ans }\end{array}$ & $\begin{array}{l}\text { Hommes } \\
20-49 \text { ans }\end{array}$ & $\begin{array}{c}\text { Femmes } \\
25-39 \text { ans }^{(a)}\end{array}$ & $\begin{array}{l}\text { Hommes } \\
30-44 \text { ans }^{(a)}\end{array}$ \\
\hline Équateur & 620 & 519 & 536 & 441 \\
\hline Colombie & 522 & 278 & 463 & 226 \\
\hline Pérou et Bolivie & 422 & 285 & 365 & 234 \\
\hline Argentine, Uruguay et Chili & 413 & 403 & 361 & 340 \\
\hline Autres pays d'Amérique latine & 793 & 472 & 692 & 398 \\
\hline Maroc & 574 & 760 & 506 & 651 \\
\hline Autres pays d'Afrique & 184 & 415 & 168 & 375 \\
\hline Europe occidentale $\mathrm{e}^{(\mathrm{b})}$ & 1180 & 1157 & 1024 & 1048 \\
\hline Roumanie & 632 & 557 & 555 & 444 \\
\hline Autres pays d'Europe ${ }^{(c)}$ & 515 & 342 & 461 & 288 \\
\hline Asie & 115 & 204 & 95 & 174 \\
\hline Total & 5970 & 5392 & 5226 & 4619 \\
\hline \multicolumn{5}{|c|}{$\begin{array}{l}\text { (a) Individus ayant appartenu aux groupes d'âges étudiés pendant les trois années précédant l'entretien. } \\
\text { (b) Allemagne, Andorre, Autriche, Belgique, Danemark, Espagne, Finlande, France, Grèce, Irlande, Islande, Italie, } \\
\text { Liechtenstein, Luxembourg, Malte, Monaco, Norvège, Pays-Bas, Portugal, Royaume-Uni, Suède, Suisse. Le Canada, } \\
\text { les États-Unis, I'Australie et la Nouvelle-Zélande sont inclus dans ce groupe car ils ne représentent que } 4 \text { \% des } \\
\text { immigrés. } \\
\text { (c) Albanie, Biélorussie, Bosnie-Herzégovine, Bulgarie, Croatie, Estonie, Hongrie, Lettonie, Lituanie, Moldavie, } \\
\text { Monténégro, Pologne, République tchèque, Russie, Serbie, Slovaquie, Slovénie, Ukraine. } \\
\text { Source : ENI (2007). }\end{array}$} \\
\hline
\end{tabular}

1. Les enfants résidant avec l'enquêté(e) à partir des tableaux par ménage de l'ENI, qui contiennent les informations sur l'âge, l'année et le pays de naissance de ces enfants, ainsi qu'une matrice des relations de parenté entre les membres du ménage.

2. Les enfants vivants qui ne résident pas avec l'enquêté(e) à partir des tableaux de l'ENI concernant ces enfants, qui fournissent l'information sur leur âge et leur pays de naissance.

3. Les enfants décédés (4\% des enfants vivants et décédés). L'ENI fournit une information sur le nombre d'enfants décédés, mais pas leurs dates de naissance. Nous avons imputé celles-ci en générant des nombres aléatoires et en utilisant l'information sur l'âge de l'enquêté(e) et un profil simplifié de fécondité par âge ${ }^{(10)}$. Ainsi, pour un(e) enquêté(e) âgé(e) de 20 ans, la date de naissance de l'enfant décédé doit être dans la période 2002-2006 ; pour une femme âgée de 60 ans, l'année de naissance la plus vraisemblable de l'enfant décédé se situe dans la période 1967-1981, lorsque sa mère était aux âges de fécondité maximale (l'année de naissance ne peut se situer ni avant 1962 ni après 1992,

(10) Le profil de fécondité postule d'une part que la fécondité est nulle avant 15 ans et à partir de 45 ans, d'autre part que la fécondité à 15-18 ans et 33-44 ans est deux fois moindre qu'à 19-32 ans. 
hors des âges féconds). Des 1088 enfants décédés, seuls 33 sont le résultat d'une imputation qui situe leur naissance dans la période 2004-2006 retenue ici, 29 d'entre eux étant nés en Espagne après la migration.

Seuls les enfants nés en Espagne ont été inclus dans le calcul des taux de fécondité et seul le temps passé en Espagne a été retenu pour la population figurant au dénominateur. Par exemple, un immigré fêtant son $30^{\mathrm{e}}$ anniversaire le jour de l'entretien et qui était arrivé 18 mois plus tôt est comptabilisé pour une demi-année d'exposition au risque de fécondité à l'âge de 28 ans, une année pleine à 29 ans et zéro à tous les autres âges.

Nous avons obtenu une estimation centrale et un intervalle de confiance pour l'indicateur conjoncturel de fécondité (ICF, somme des taux de fécondité par année d'âge), ainsi que pour le nombre d'immigrés à partir de la formule ci-dessus. Les intervalles de confiance à $95 \%$ (IC) ont été estimés par la procédure du bootstrap (Efron et Tibshirani, 1993 ; Poi, 2004), en simulant 500 fois le tirage d'échantillons de 11600 individus pour l'indicateur conjoncturel de fécondité et de 9100 individus pour les effectifs d'immigrés. Les centiles 2,5 et 97,5 de la distribution des résultats pour les 500 échantillons simulés ont fourni des estimations des IC à $95 \%$. On notera que les IC relatifs ainsi estimés sont légèrement supérieurs à ceux qu'on obtiendrait pour la fécondité avec une distribution de Poisson.

\section{Résultats}

\section{La fécondité des immigrés}

Sur la figure 1 est représenté l'indicateur conjoncturel de fécondité pour les hommes et les femmes, calculé à partir des taux de fécondité par année d'âge tirés de l'ENI, et l'IC à 95 \% estimé par rééchantillonnage (bootstrapping). Les niveaux de fécondité sont bas pour presque tous les groupes d'immigrés. Comme dans des études antérieures (Castro-Martín et Rosero-Bixby, 2011), seuls les hommes et femmes du Maroc et les femmes venues d'autres pays africains - essentiellement d'Afrique subsaharienne - ont des taux de fécondité supérieurs au niveau de remplacement. Dans les autres groupes, la fécondité des femmes se situe en moyenne entre 1,4 naissance pour les Roumaines et 1,6 pour les femmes d'Europe occidentale et d'Amérique latine. Ces niveaux sont supérieurs à ceux des femmes nées en Espagne (1,26 naissance pour la même période), mais inférieurs à ceux enregistrés dans la plupart des pays d'origine. On trouvera une analyse approfondie de la fécondité des immigrés d'après l'ENI dans Castro-Martín et Rosero-Bixby (2011).

La fécondité des hommes immigrés est inférieure à celle des femmes, sauf pour ceux originaires d'Équateur. L'écart est important pour les Marocains et les autres Africains, groupes qui présentent aussi le plus fort déséquilibre entre les effectifs d'hommes et de femmes immigrés (selon le registre de population 
en 2007, les rapports de masculinité correspondant aux âges féconds sont de 2,5 et 3,3 alors qu'ils sont inférieurs à 1,3 dans les autres groupes). La fécondité particulièrement faible des hommes arrivés d'Afrique pourrait être due à la pénurie de femmes de même origine, au faible taux de mariages mixtes et à la lenteur du regroupement familial. La situation n'est cependant pas symétrique concernant la fécondité des femmes. La relative pénurie d'hommes de même origine (les originaires des autres pays d'Amérique latine ont un rapport de masculinité de 0,7 ) ne se traduit pas par une fécondité féminine inférieure aux attentes. La moindre fécondité des hommes provient peut-être d'une sous-déclaration des enfants dans l'ENI, car ce défaut est souvent signalé dans la littérature démographique (Rendall et al., 1999). La relation inverse avec le rapport de masculinité des adultes suggère cependant que l'écart de fécondité entre hommes et femmes ne tient pas seulement à une mauvaise déclaration des naissances. Les hommes venus d'Amérique latine et du Maroc ont un ICF nettement supérieur aux hommes nés en Espagne. Pour les autres hommes immigrés, leur fécondité ne diffère pas de celle des Espagnols de manière significative.

Figure 1. Indicateur conjoncturel de fécondité (ICF) par sexe pour les Espagnols et les immigrés par pays ou région de naissance, 2004-2006

Femmes

Hommes

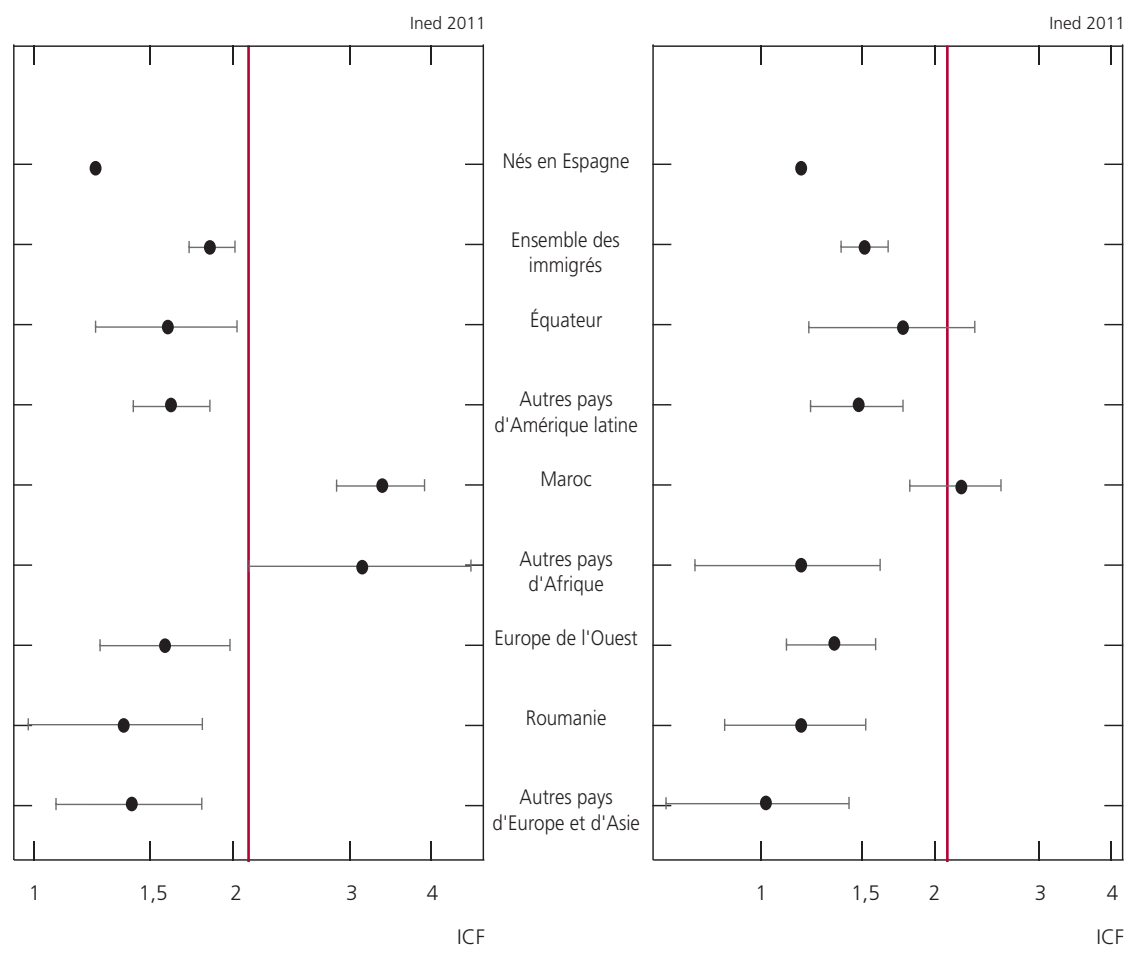

Note : Les estimations de l'ICF et les intervalles de confiance

à $95 \%$ sont représentés sur une échelle logarithmique.

Sources: Immigrés : ENI (2007); Nés en Espagne : État civil. 
Figure 2. Taux de fécondité par âge des immigrés et des Espagnols par sexe, 2004-2006
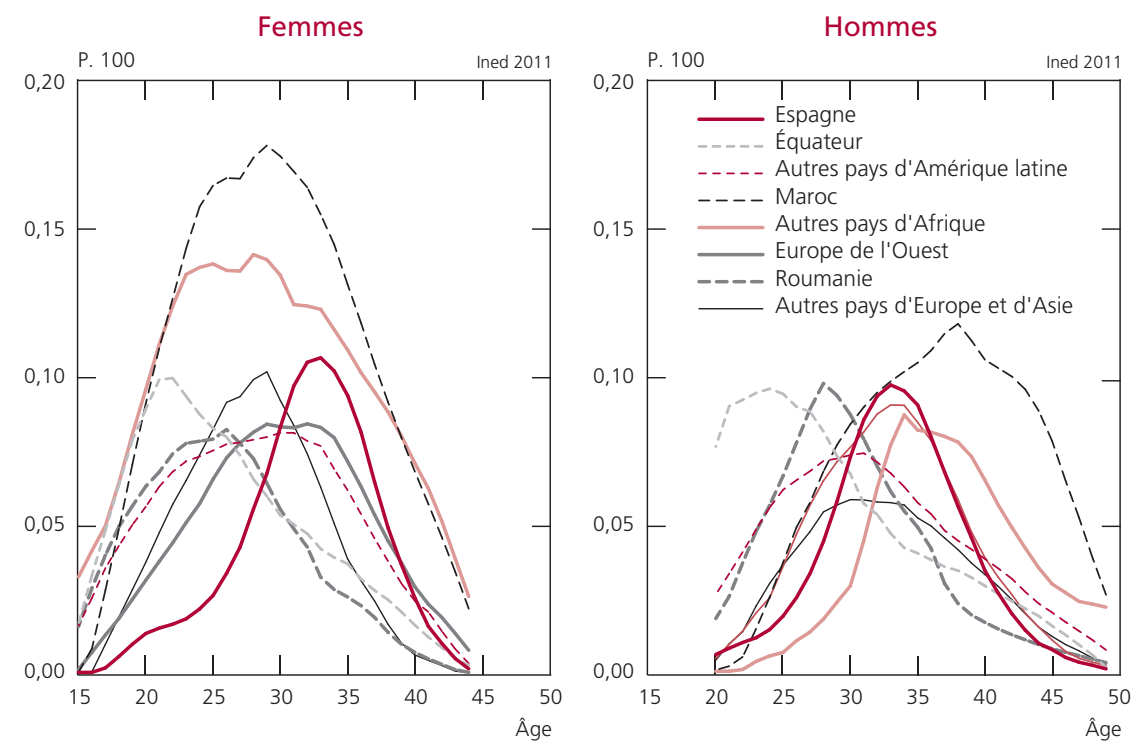

Sources : Immigrés : ENI (2007) ; Nés en Espagne : État civil.

Sur la figure 2, on a représenté les courbes lissées ${ }^{(11)}$ des taux de fécondité par âge obtenus à partir de l'enquête ENI pour les sept groupes d'immigrés. À titre de comparaison, on a fait figurer aussi la courbe de fécondité de 2005 pour les personnes nées en Espagne. Chez les femmes, nous distinguons quatre profils de fécondité par âge :

- Les Espagnoles (groupe de référence) ont une fécondité tardive,

- Les Européennes occidentales ont un profil de fécondité semblable à celui des Espagnoles après 30 ans et des taux légèrement plus élevés avant cet âge.

- Les immigrées africaines (y compris les Marocaines) ont une fécondité nettement plus élevée et plus précoce.

- Les femmes des autres nationalités (autres pays d'Amérique latine et d'Europe) ont une fécondité plus élevée que les Espagnoles aux jeunes âges et plus faible à partir d'environ 30 ans.

Pour les hommes, on distingue cinq profils de fécondité selon l'âge :

- Les Espagnols et les Européens de l'Ouest.

- Les Marocains ont une fécondité plus forte que les Espagnols à tous les âges, surtout après 35 ans.

- Les autres Africains ont une fécondité moindre que les Espagnols jusque vers 35 ans, et plus élevée après cet âge.

(11) Les courbes de fécondité selon l'âge ont été lissées par des régressions locales (procédure Lowess du logiciel Stata, avec une utilisation locale de l'échantillon (bandwidth) de 0,25). 
- Les Équatoriens et, dans une certaine mesure, les Roumains et les immigrés en provenance des autres pays d'Europe et d'Asie ont une fécondité précoce nettement supérieure à celle des Espagnols et une fécondité tardive inférieure.

- Les originaires d'autres pays d'Amérique latine ont des taux de fécondité relativement élevés après 40 ans et des taux compris entre ceux de l'Espagne et de l'Équateur aux jeunes âges.

En général, les femmes immigrées ont un ICF un peu plus élevé que les femmes nées en Espagne : 1,86 naissance (IC à $95 \%$ [1,72-2,02]) contre 1,26 ; leur âge moyen à la maternité est nettement plus précoce : 28,8 ans contre 32,3 ans pour les Espagnoles. Les hommes immigrés ont un ICF plus élevé que leurs homologues nés en Espagne : 1,52 naissance (IC à $95 \%$ [1,38-1,66]) contre 1,19 ; l'écart est cependant moindre que pour les femmes. Le profil de la fécondité par âge des hommes immigrés (âge moyen de fécondité : 33,6 ans) diffère peu de celui des Espagnols (34,0 ans), à l'exception des Équatoriens qui sont pères nettement plus jeunes.

\section{Le nombre estimé d'immigrés}

Les nombres estimés d'immigrés aux âges féconds (tableau 2) sont obtenus en divisant par les taux de fécondité selon l'âge les nombres d'enfants nés de mères ou pères immigrés classés par âge dans l'état civil espagnol (annexe). Les taux de fécondité sont relatifs à la période 2004-2006 et les naissances enregistrées à l'année 2007, la première à laquelle est disponible l'information sur le pays de naissance des parents. L'estimation du nombre d'immigrés est donc

Tableau 2. Nombre d'immigrés en Espagne mi-2007

dans le Registre de population municipal et estimés à partir des statistiques de naissances et des taux de fécondité

\begin{tabular}{|c|c|c|c|c|c|c|}
\hline \multirow{3}{*}{$\begin{array}{l}\text { Pays de } \\
\text { naissance }\end{array}$} & \multicolumn{4}{|c|}{ Nombre d'immigrés (milliers) } & \multirow{2}{*}{\multicolumn{2}{|c|}{$\begin{array}{c}\text { Rapport } \\
\text { Registre/Estimation }\end{array}$}} \\
\hline & \multicolumn{2}{|c|}{ Femmes 20-39 ans } & \multicolumn{2}{|c|}{ Hommes 25-44 ans } & & \\
\hline & Registre & Estimation & Registre & Estimation & Femmes & Hommes \\
\hline Équateur & 126,4 & 159,7 & 121,0 & 145,0 & 0,79 & 0,83 \\
\hline $\begin{array}{l}\text { Reste de } \\
\text { l'Amérique latine }\end{array}$ & 488,0 & 405,3 & 402,0 & 346,6 & 1,20 & 1,16 \\
\hline Maroc & 113,1 & 114,7 & 237,6 & 189,9 & 0,99 & 1,25 \\
\hline Afrique & 41,6 & 42,0 & 127,4 & 147,0 & 0,99 & 0,87 \\
\hline $\begin{array}{l}\text { Europe } \\
\text { occidentale }\end{array}$ & 203,8 & 150,7 & 270,6 & 214,9 & 1,35 & 1,26 \\
\hline Roumanie & 178,5 & 193,5 & 188,7 & 140,8 & 0,92 & 1,34 \\
\hline EurAsie & 171,1 & 204,5 & 219,4 & 179,3 & 0,84 & 1,22 \\
\hline Total & 1322,5 & 1270,5 & 1566,8 & 1363,5 & 1,04 & 1,15 \\
\hline
\end{tabular}


calculée pour mi-2007. La date de référence étant le ${ }^{\text {er }}$ janvier de chaque année pour le registre de population, la comparaison porte sur la moyenne des résultats de 2007 et 2008. Sur la figure 3, on a représenté les rapports du nombre d'immigrés dans le registre à celui estimé par calcul et leur intervalle de confiance à $95 \%$. Des rapports égaux à 1,0 et des intervalles de confiance englobant 1,0 marquent l'absence de différence significative entre les deux nombres.

Le nombre de femmes immigrées du registre de population est presque égal à notre estimation d'après les données sur les naissances et les taux de fécondité (tableau 2 et figure 3) : le rapport est 1,04 (IC à $95 \%$ [0,96-1,13]). Cependant, le nombre d'hommes immigrés dans le registre dépasse notre estimation de $15 \%$ (IC à $95 \%$ [1,04-1,26]).

Figure 3. Ratio des nombres d'immigrés

(registre de population/estimation) en Espagne, 2007.

Femmes (20-39 ans)

Hommes (25-44 ans)

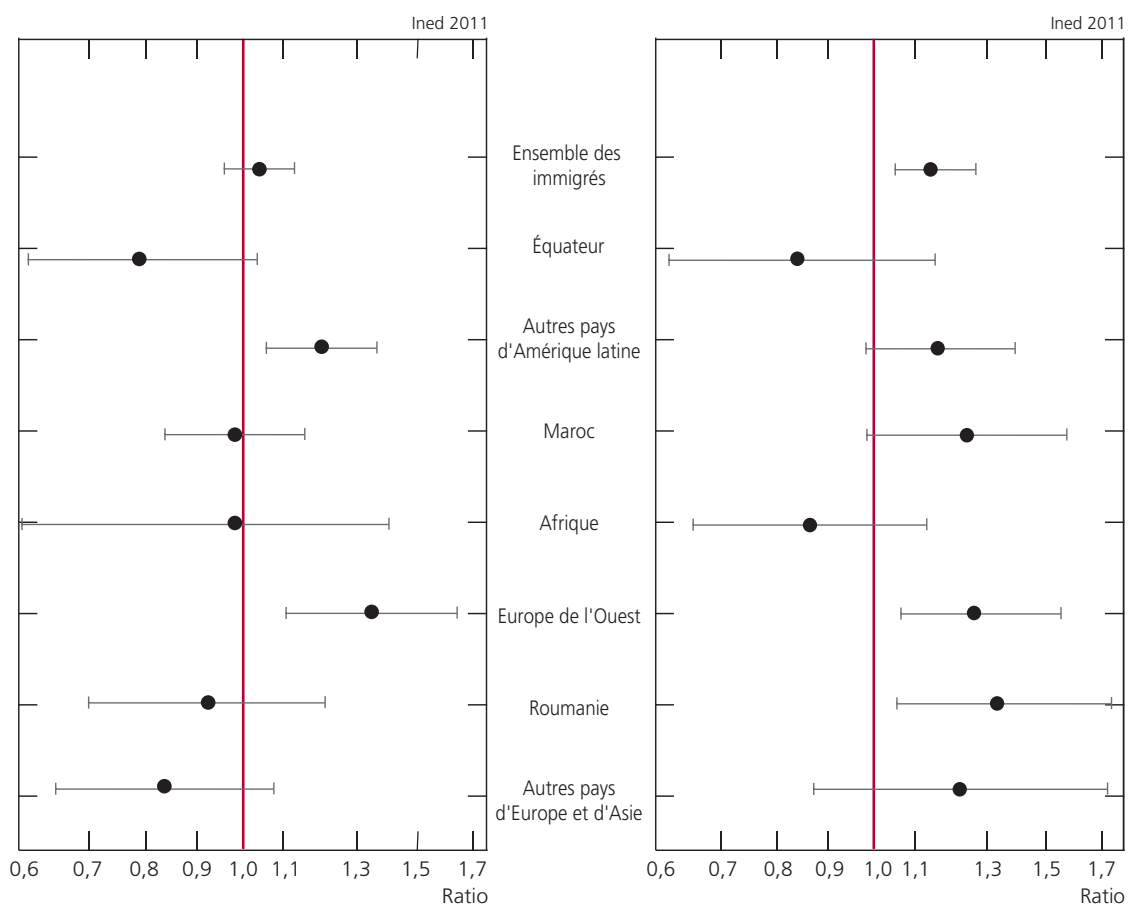

Note : Le ratio et les intervalles de confiance à $95 \%$ sont représentés sur une échelle logarithmique. Sources: ENI (2007); État civil.

Les femmes venues d'Europe occidentale (rapport : 1,35) et d'Amérique latine hors Équateur $(1,20)$ sont significativement plus nombreuses dans le registre de population que dans notre estimation. Il est possible que certaines Européennes retournent dans leur pays pour accoucher et que la naissance de leurs enfants ne soit donc pas enregistrée par l'état civil espagnol ; il n'y aurait 
donc pas nécessairement surenregistrement des Européennes occidentales au niveau municipal. Pour les hommes, le décompte du registre excède l'estimation dans tous les groupes (sauf les immigrés venus d'Équateur et d'Afrique subsaharienne) ; toutefois, l'écart n'est statistiquement significatif au seuil de $5 \%$ que pour ceux venus d'Europe occidentale et de Roumanie.

\section{Discussion}

Nous avons présenté une méthode indirecte simple d'estimation du nombre d'immigrés aux âges féconds à partir d'informations sur les enfants classés par origine de leurs parents et sur les taux de fécondité des immigrés. La méthode, déjà utilisée avec succès pour estimer le nombre de Nicaraguayens au Costa Rica (Rosero-Bixby et al., 2002), a été appliquée aux données espagnoles de 2007. Nos résultats diffèrent selon le sexe. Pour les femmes, nos résultats valident à la fois le décompte du registre de population en 2007 et la méthode indirecte d'estimation du nombre d'immigrées : les deux résultats sont à peu près les mêmes. En revanche, le registre décompte $15 \%$ d'hommes immigrés de plus que notre estimation, avec une différence statistiquement significative.

Cet écart sur le nombre d'hommes immigrés pourrait être interprété comme une surévaluation du nombre d'immigrés par le registre de population (Ródenas et Martí, 2009). Bien que les procédures destinées à éviter le double enregistrement et à détecter les départs d'Espagne aient été renforcées depuis 2006, il subsiste sans doute un surdénombrement en 2007. Une explication complémentaire serait que nos estimations du nombre d'immigrés sont sous-évaluées, du fait que les estimations de fécondité à partir de l'ENI ont tendance à être surévaluées, comme c'est souvent le cas dans les enquêtes (Festy et Prioux, 2002). Mais il serait étonnant que ce biais dans la mesure de la fécondité n'affecte que les hommes. En outre, le fait que l'ENI fournisse des ICF très bas pour les hommes entrerait en contradiction avec une hypothèse de surestimation des niveaux de fécondité. Néanmoins, la qualité des données sur la fécondité masculine est un sujet peu abordé par les études démographiques et c'est pourquoi nos estimations sont sans doute moins robustes pour les hommes que pour les femmes.

Une manière indirecte d'apprécier l'écart entre les estimations d'hommes immigrés est de comparer le rapport de masculinité dans diverses sources. S'il y avait surdénombrement des hommes immigrés par le registre de population, le rapport de masculinité y serait plus élevé que dans d'autres sources. C'est bien ce que montre le tableau 3. Le rapport de masculinité des immigrants dans le registre de population est beaucoup plus élevé que ceux issus de l'échantillon non pondéré de l'enquête nationale sur les immigrants (ENI) et de l'enquête sur la participation à l'emploi (EPA) réalisée chaque trimestre sur un échantillon de presque 200000 personnes. Après pondération, l'ENI fournit pour les âges considérés un rapport de masculinité proche de celui du registre, 
mais c'est parce que l'échantillon de cette enquête est post-stratifié précisément pour que sa structure par sexe soit identique à celle du registre, que nous supposons être biaisée.

Tableau 3. Rapport de masculinité des immigrés selon le registre municipal de population, l'enquête nationale sur les immigrés (ENI) et l'enquête sur l'emploi (EPA)

\begin{tabular}{|l|c|}
\hline \multicolumn{1}{|c|}{ Sources des données } & Rapport de masculinité \\
\hline 2005-2006 Registre municipal de population, 20-49 ans & 1,22 \\
2007-2008 Registre municipal de population, 20-49 ans & 1,19 \\
2006-2007 ENI, 20-49 ans, pondéré & 1,20 \\
2006-2007 ENI, 20-49 ans, non pondéré & 0,90 \\
2005-2006 EPA, 16-44 ans & 1,02 \\
\hline
\end{tabular}

Les hommes et femmes venus d'Europe occidentale (en majorité de France, du Portugal et d'Allemagne) ainsi que les hommes venus de Roumanie sont les groupes pour lesquels le rapport est le plus élevé entre les nombres d'immigrés enregistré et estimé. Ce résultat peut être rapproché du mode de résidence transnational de certaines de ces personnes, qui est facilité par le fait qu'elles sont citoyennes de l'Union européenne et bénéficient du droit de vivre et de travailler en Espagne sans avoir à demander d'autorisation de résidence et de travail. On doit aussi avoir à l'esprit que les immigrés originaires de l'Union européenne n'ont pas été concernés par la réforme légale exigeant un réenregistrement tous les deux ans, et qu'ils présentent de ce fait un risque plus important que les autres d'être encore dans le registre alors qu'ils ne résident plus en Espagne de manière continue. Nos résultats révèlent peut-être aussi l'existence d'une importante migration temporaire ou saisonnière, en particulier de la part des Roumains. La relative proximité des pays européens rend possibles ces stratégies. En outre, si certains Européens résidant en Espagne de manière temporaire ou permanente décident d'avoir et d'enregistrer leurs enfants dans leur pays d'origine, nos estimations du nombre d'immigrés seront sous-évaluées et inférieures au décompte du registre de population. En fait, ni le registre de population ni nos estimations de la population immigrée à partir de la fécondité ne peuvent saisir une réalité aussi complexe.

Le schéma inverse, dans lequel le ratio entre population dans le registre et nombre estimé d'immigrés est inférieur à l'unité, s'applique aux hommes et femmes venus d'Équateur et aux hommes d'Afrique, ce qui laisse supposer que le dénombrement de ces groupes dans le registre serait incomplet, bien que l'écart entre les deux sources ne soit pas statistiquement significatif.

Une limitation dans l'application à l'Espagne de la méthode proposée est que la classification des naissances par pays de naissance des parents n'est disponible qu'à partir de 2007, alors que les estimations de fécondité à partir 
de l'enquête ENI 2007 correspondent à la période 2004-2006. Nous supposons donc que la fécondité des immigrés n'a pas varié significativement en deux ans. Si par exemple l'ICF des immigrés avait diminué de $10 \%$ en deux ans, nous sous-estimerions le nombre d'immigrés de $10 \%$ et ce serait une autre explication possible du décompte plus élevé des hommes immigrés dans le registre de population. Il n'y a cependant pas de signes montrant que les taux de fécondité des immigrés aient baissé au cours de cette période. Selon les estimations de l'Institut national de statistiques, l'ICF des étrangers s'est même légèrement accru, passant de 1,34 en 2005 à 1,39 en 2007.

Malgré toutes les réserves mentionnées, la méthode indirecte proposée dans cet article apparaît comme un instrument utile pour estimer le nombre d'immigrés et pour valider les décomptes du registre de population. Les résultats obtenus suggèrent que le nombre de femmes immigrées est correctement mesuré par le registre, mais que le nombre d'hommes est surévalué (d'environ $15 \%$, avec une marge d'incertitude de $4 \%$ à $26 \%$ ). Ces conclusions valent pour 2007, et il est possible que le surenregistrement ait ensuite diminué grâce à la systématisation de la procédure de radiation du registre des immigrés n'ayant pas renouvelé leur inscription au bout de deux ans. Au-delà, le souci de soumettre le registre de population à la validation par confrontation avec des sources externes doit s'imposer de manière permanente.

Remerciements : Une version antérieure de cet article a été présentée au XXVI ${ }^{e}$ Congrès international de la population, Marrakech, 27 septembre - 2 octobre 2009. Cette recherche a bénéficié du soutien financier du ministère espagnol de la Science et de l'innovation, dans le cadre des projets CSO2008-03616/SOCI et CSO2009-11883/SOCI. Nous remercions également Andreu Domingo et les évaluateurs de la revue pour leurs utiles commentaires. 


\section{ANNEXE}

Taux de fécondité par âge des immigrés en Espagne (2004-2006) selon I'ENI, et naissances issues de parents immigrés (État civil)

\begin{tabular}{|c|c|c|c|c|c|c|c|}
\hline & \multicolumn{7}{|c|}{ Pays de naissance des immigrés } \\
\hline & Équateur & $\begin{array}{l}\text { Autre pays } \\
\text { d'Amérique } \\
\text { latine }\end{array}$ & Maroc & $\begin{array}{l}\text { Autres pays } \\
\text { d'Afrique }\end{array}$ & $\begin{array}{l}\text { Europe de } \\
\text { L'Ouest }\end{array}$ & Roumanie & $\begin{array}{c}\text { Autres pays } \\
\text { d'Europe et } \\
\text { d'Asie }\end{array}$ \\
\hline \multicolumn{8}{|c|}{ Taux de fécondité } \\
\hline \multicolumn{8}{|c|}{ Femmes } \\
\hline $20-24$ ans & 0,118 & 0,087 & 0,139 & 0,175 & 0,043 & 0,061 & 0,064 \\
\hline $25-29$ ans & 0,056 & 0,075 & 0,202 & 0,123 & 0,086 & 0,084 & 0,104 \\
\hline $30-34$ ans & 0,056 & 0,084 & 0,162 & 0,131 & 0,094 & 0,041 & 0,085 \\
\hline $35-39$ ans & 0,033 & 0,050 & 0,128 & 0,139 & 0,058 & 0,019 & 0,018 \\
\hline \multicolumn{8}{|l|}{ Hommes } \\
\hline $25-29$ ans & 0,090 & 0,071 & 0,064 & 0,011 & 0,053 & 0,092 & 0,061 \\
\hline 30-34 ans & 0,042 & 0,072 & 0,091 & 0,069 & 0,108 & 0,080 & 0,056 \\
\hline 35-39 ans & 0,042 & 0,058 & 0,112 & 0,091 & 0,070 & 0,024 & 0,059 \\
\hline $40-44$ ans & 0,028 & 0,030 & 0,149 & 0,046 & 0,024 & 0,020 & 0,023 \\
\hline \multicolumn{8}{|c|}{ Nombre de naissances issues de } \\
\hline \multicolumn{8}{|c|}{ Mères immigrées } \\
\hline $20-24$ ans & 1846 & 6444 & 5231 & 1049 & 834 & 3593 & 2577 \\
\hline $25-29$ ans & 3176 & 9451 & 5844 & 2134 & 2037 & 3895 & 4658 \\
\hline 30-34 ans & 2592 & 8938 & 4397 & 1733 & 4561 & 2141 & 3555 \\
\hline $35-39$ ans & 1356 & 4942 & 2692 & 750 & 3428 & 684 & 1397 \\
\hline \multicolumn{8}{|c|}{ Pères immigrés } \\
\hline $25-29$ ans & 2881 & 6647 & 3383 & 850 & 1524 & 3727 & 3108 \\
\hline 30-34 ans & 2495 & 7130 & 5985 & 1795 & 4608 & 3083 & 3491 \\
\hline $35-39$ ans & 1362 & 4849 & 5638 & 1786 & 4420 & 1214 & 1954 \\
\hline $40-44$ ans & 593 & 2109 & 3126 & 1106 & 1928 & 223 & 757 \\
\hline
\end{tabular}




\section{RÉFÉRENCES}

ARANGO J., 2000, « Becoming a country of immigration at the end of the twentieth century: The case of Spain », in King R., Lazaridis G., Tsardanidis C. (eds.), Eldorado or Fortress? Migration in Southern Europe, London, Macmillan, p. 253-276.

BEAN F. D., King A. G., PASSEL J. S., 1983, « The number of illegal migrants of Mexican origin in the United States: Sex ratio-based estimates for 1980 », Demography, 20(1), p. 99-109.

BORJAS G. J., FREEMAN R. B., LANG K., 1991, « Undocumented Mexican-born workers in the United States: How many, how permanent? », in Abowd J. M., Freeman R. B. (eds.), Immigration, Trade, and the Labor Market, Chicago, National Bureau of Economic Research, The University of Chicago Press, p. 77-100.

CAstro-Martín T., Rosero-Bixby L., 2011, « Maternidades y fronteras. La fecundidad de las mujeres inmigrantes en España », in Reher D., Requena M., Sanz-Gimeno A. (eds.), La inmigración en España: perspectivas innovadoras, numéro spécial de la Revista Internacional de Sociología, 69(M1), p. 105-138,

http://revintsociologia.revistas.csic.es/index.php/revintsociologia/article/view/388/398

Cebolla H., GONZÁlez-Ferrer A., 2008, La inmigración en España (2000-2007). De la gestión de flujos a la integración de los inmigrantes, Madrid, Centro de Estudios Políticos y Constitucionales, 318 p.

Devolver D., Domingo A., GArCía J., 2003, Fecundidad diferencial y potencial de reagrupación familiar de la población extranjera de la Comunidad de Madrid a partir del Padrón continuo a 1/1/1999, Bellaterra, Centre d'Estudis Demogràfics, Article de Demografía $\mathrm{n}^{\circ} 224,32$ p., http://www.ced.uab.es/publicacions/ArticlesPDF/Text224.pdf

EFron B., TibShIRANi R. J., 1993, An Introduction to the Bootstrap, New York, Chapman $\&$ Hall/CRC, $443 \mathrm{p}$.

EUROSTAT, 2009, L'Europe en chiffres. L'annuaire d'Eurostat 2009, Luxembourg, Commission européenne, 566 p.,

http://epp.eurostat.ec.europa.eu/portal/page/portal/product_details/publication?p_ product_code=KS-CD-09-001

FESTy P., PRIOUX F., 2002, An Evaluation of the Fertility and Family Surveys Project, Genève, Nations unies, United Nations Publication number GV.E.01.II.E.23, 74 p.

GONZÁLEZ-ENRÍQUEZ C., 2009, « Spain, the cheap model. Irregularity and regularisation as immigration management policies », European Journal of Migration and Law, 11(2), p. 139-157.

GONZÁleZ-FERRER A., 2009, Country Report Spain, Promoting Comparative Quantitative Research in the Field of Migration and Integration in Europe (PROMINSTAT), 26 p., http://ec.europa.eu/ewsi/UDRW/images/items/docl_9019_550544131.pdf

GonzÁlez-Ferrer A., Cortina C., 2011, Naturalization decisions in Spain. The importance of legal asymmetries, Paper presented at the Annual Meeting of the Population Association of America, Washington DC, March 31 - April 2, 2011, http://paa2011.princeton.edu/download.aspx?submissionId=112356

INSTITUTO NACIONAL DE ESTADÍSTICA (INE), 2008, Metodología general de la Encuesta Nacional de Inmigrantes, 34 p.,

http://www.ine.es/en/daco/daco42/inmigrantes/inmigra_meto_en.pdf 
INSTITUTO NACIONAL DE ESTADÍSTICA (INE), 2009, Encuesta Nacional de Inmigrantes 2007: una monografía, http://www.ine.es/prodyser/pubweb/eni07/eni07.htm

IZQUIERDO A., LÓPEZ DE LERA D. (eds.), 2006, Demografía de los extranjeros: incidencia en el crecimiento de la población, Bilbao, Fundación Banco Bilbao Vizcaya Argentaria, $333 \mathrm{p}$.

MARTIN P., 2009, « Recession and migration: A new era for labor migration? », International Migration Review, 43(3), p. 671-691.

MOREnO FUENTES F. J., 2005, The Regularisation of Undocumented Migrants as a Mechanism for the 'Emerging' of the Spanish Underground Economy, CSIC, Unidad de Políticas Comparadas, Working Article 05-06, http://www.ipp.csic.es/en/content/the_regularisation_of_undocume

Muller T., EsPenshade T. J., 1985, The Fourth Wave: California's Newest Immigrants, Washington, D.C., The Urban Institute Press.

POI B. P., 2004, «From the help desk: Some bootstrapping techniques », Stata Journal, 4(3), p. 312-328.

REHER D., SILVESTRE J., 2009, «Internal migration patterns of foreign-born immigrants in a country of recent mass immigration: Evidence from new micro data for Spain », International Migration Review, 43(4), p. 815-849.

REHER D., REQUENA M., 2009a, « The National Immigrant Survey of Spain: A new data source for migration studies in Europe ». Demographic Research, 20(12), p. 253-278, http://www.demographic-research.org/Volumes/Vol20/12/20-12.pdf

REHER D., REQUENA M. (eds.), 2009b, Las múltiples caras de la inmigración en España, Madrid, Alianza Editorial, 321 p.

REHER D., REQUENA M., SANZ-GIMENO A. (eds.), 2011a, La inmigración en España: perspectivas innovadoras, numéro spécial de la Revista Internacional de Sociología, 69(M1), 292 p.,

http://revintsociologia.revistas.csic.es/index.php/revintsociologia/article/view/385/395

REHER D., REQUENA M., SANZ-GIMENO A., 2011b, « ¿España en la encrucijada? Consideraciones sobre el cambio de ciclo migratorio », in Reher D., Requena M., Sanz-Gimeno A. (eds.), La inmigración en España: perspectivas innovadoras, numéro spécial de la Revista Internacional de Sociología, 69(M1), p. 9-44,

http://revintsociologia.revistas.csic.es/index.php/revintsociologia/issue/view/29

Rendall M. S., Clarke L., Peters E., Ranjit N., Verropoulou G., 1999, « Incomplete reporting of men's fertility in the United States and Britain: A research note », Demography, 36(1), p. 135-144.

ROBINSON J. G., 1980, « Estimating the approximate size of the illegal alien population in the United States by the comparative trend analysis of age-specific death rates », Demography, 17(2), p. 159-176.

ROIG Vila M., CASTRO MARTíN T., 2007, « La fécondité des étrangères dans un pays d'immigration récente : le cas de l'Espagne », Population, 62(3), p. 419-450.

Ródenas Calatayud C., Martí Sempere M., 2009, « L'estimation des “fausses migrations" en Espagne », Population, 64(2), p. 397-412.

Rosero-Bixby L., Brenes G., Chen M., 2002, « Fecundidad diferencial ee inmigrantes nicaragüenses en Costa Rica », Notas de Población, 74, p. 27-52, http://www.eclac.org/publicaciones/xml/6/11066/lcg2148p_3.pdf

STATACORP, 2007, Stata Statistical Software: Release 10, Texas, College Station, Stata Corporation.

NATIONS UNIES, 1983, Manual X: Indirect Techniques for Demographic Estimation, New York, United Nations, Department of International Economic and Social Affairs.

ZABA B., 1985, Estimación de la emigración mediante la utilización de técnicas indirectas, Liège, Ordina Editors/IUSSP, 170 p. 
Luis Rosero-Bixby, Teresa CAStro-Martín, David Reher, María SÁnChez-

DOMÍNGUEZ • ESTIMATION INDIRECTE DU NOMBRE D'IMMIGRÉS EN ESPAGNE À PARTIR DES TAUX DE FÉCONDITÉ ET DES NAISSANCES

Cet article propose une méthode indirecte pour valider les décomptes du nombre d'immigrés en Espagne établis à partir des registres municipaux de population, qui pourraient surestimer le nombre d'immigrés du fait de doubles enregistrements et de défauts de radiation en cas de départ du pays. La méthode proposée utilise deux types d'informations : le nombre de naissances issues d'immigrés et leurs taux de fécondité. Les statistiques de naissances par origine des parents proviennent de l'état civil espagnol ; les taux de fécondité sont estimés à partir de l'Enquête nationale sur les immigrés de 2007. Pour les femmes, l'estimation indirecte ne diffère pas significativement du décompte dans le registre, ce qu'on peut considérer comme une validation des deux sources. Pour les hommes, le registre de population dénombre $15 \%$ d'immigrés de plus que l'estimation indirecte, avec un écart statistiquement significatif. Comparés aux estimations proposées dans cet article, les dénombrements des hommes et femmes provenant d'Europe occidentale et des hommes de Roumanie présentent un excédent important. À l'inverse, le rapport est faiblement inférieur à 1 pour les hommes et femmes d'Équateur et les hommes d'Afrique, ce qui suggère un sous-enregistrement de ces groupes par le registre de population.

\section{Luis Rosero-Bixby, Teresa Castro-Martín, David Reher, María SÁnchez-

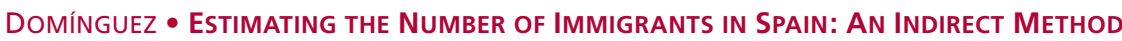 BASED ON BIRTHS AND FERTILITY RATES}

This article proposes an indirect method to validate existing estimates of immigrants' stock from the Spanish municipal population register, which some believe might be over-counting immigrants who double register in different municipalities or fail to deregister when leaving the country. The proposed method uses two pieces of information: births to immigrants and their fertility rates. Data on births by parents' origin come from the Spanish birth registry; fertility rates are estimated with data from the 2007 National Immigrant Survey. For female immigrants, the indirect estimate does not differ significantly from the count in the register, which can be taken as a validation of both sources. Among men, however, the population register counts $15 \%$ more immigrants than the indirect estimate. Western European men and women, and Romanian men are immigrant groups with substantial and statistically significant excess count in the population register compared to this article's estimate. The opposite pattern, i.e. ratio slightly below one, is found for Ecuadorian men and women and African men, suggesting that these groups might be under-counted in the population register.

\section{Luis Rosero-Bixby, Teresa CAstro-MARtín, David Reher, María SÁnchez- \\ DOMÍNGUEZ • ESTIMACIÓN INDIRECTA DEL NÚMERO DE INMIGRANTES EN ESPAÑA A PARTIR DE LAS TASAS DE FECUNDIDAD Y DE LOS NACIMIENTOS.}

Este artículo propone un método de estimación indirecta para validar las cifras de inmigrantes en España establecidas a partir de los registros municipales de población, los cuales podrían sobrestimar el número de inmigrantes a causa de las inscripciones dobles y de la omisión de bajas en el registro en caso de salida del país. El método propuesto utiliza dos tipos de información: el número de nacimientos de los inmigrantes y sus tasas de fecundidad. Las estadísticas de nacimientos según el origen de los padres provienen del estado civil; las tasas de fecundidad han sido estimadas a partir de la Encuesta nacional sobre los inmigrantes de 2007. Para las mujeres, la estimación indirecta no difiere significativamente de las cifras del registro, lo que se puede considerar como una validación de las dos fuentes. Para los hombres, la evaluación del registro de población es $15 \%$ más elevada que la estimación indirecta, con une diferencia estadísticamente significativa. Comparadas con las estimaciones propuestas en este articulo, las cifras del registro para los hombres y las mujeres proviniendo de Europa occidental y para los hombres de Rumanía presentan un excedente importante. Al contrario, para los hombres y mujeres de Ecuador y para los hombres de África el registro de población da resultados ligeramente inferiores a las estimaciones indirectas, lo que sugiere un subregistro de estos grupos.

Mots-clés : Migration internationale, nombre d'immigrés, techniques d'estimation indirecte, Espagne, registre de population, enquête nationale sur les immigrés, fécondité des immigrés.

Keywords: International migration, immigrant stock, indirect estimation techniques, Spain, population register, National Immigrant Survey, immigrant fertility

Traduit par Patrick Festy. 
